\title{
Heterologous Overexpression of Poplar SnRK2 Genes Enhanced Salt Stress Tolerance in Arabidopsis thaliana
}

OPEN ACCESS

Edited by:

Heribert Hirt,

Center for Desert Agriculture,

Saudi Arabia

Reviewed by:

Lei Wang,

Institute of Botany-The Chinese

Academy of Sciences, China

Jinpeng Gao,

Washiton State University, USA

*Correspondence:

Misato Ohtani

misato@bs.naist.jp;

Qiang Zhuge

qzhuge@njfu.edu.cn

Specialty section:

This article was submitted to

Plant Physiology,

a section of the journal

Frontiers in Plant Science

Received: 03 March 2016

Accepted: 21 April 2016

Published: 09 May 2016

Citation:

Song X, YU X, Hori C, Demura T,

Ohtani $M$ and Zhuge $Q$ (2016)

Heterologous Overexpression of

Poplar SnRK2 Genes Enhanced Salt

Stress Tolerance in Arabidopsis

thaliana. Front. Plant Sci. 7:612.

doi: 10.3389/fpls.2016.00612

\author{
Xueqing Song ${ }^{1,2}$, Xiang $\mathrm{Yu}^{2}$, Chiaki Hori ${ }^{2}$, Taku Demura ${ }^{2,3}$, Misato Ohtani ${ }^{2,3 *}$ and \\ Qiang Zhuge ${ }^{1 *}$
}

${ }^{1}$ Key Laboratory of Forest Genetics and Biotechnology, Co-Innovation Center for Sustainable Forestry in Southern China, Ministry of Education, Nanjing Forestry University, Nanjing, China, ${ }^{2}$ Biomass Engineering Program Cooperation Division, RIKEN Center for Sustainable Resource Science, Yokohama, Japan, ${ }^{3}$ Graduate School of Biological Sciences, Nara Institute of Science and Technology, Ikoma, Japan

Subfamily 2 of SNF1-related protein kinase (SnRK2) plays important roles in plant abiotic stress responses as a global positive regulator of abscisic acid signaling. In the genome of the model tree Populus trichocarpa, 12 SnRK2 genes have been identified, and some are upregulated by abiotic stresses. In this study, we heterologously overexpressed the PtSnRK2 genes in Arabidopsis thaliana and found that overexpression of PtSnRK2.5 and PtSnRK2.7 genes enhanced stress tolerance. In the PtSnRK2.5 and PtSnRK2.7 overexpressors, chlorophyll content, and root elongation were maintained under salt stress conditions, leading to higher survival rates under salt stress compared with those in the wild type. Transcriptomic analysis revealed that PtSnRK2.7 overexpression affected stress-related metabolic genes, including lipid metabolism and flavonoid metabolism, even under normal growth conditions. However, the stress response genes reported to be upregulated in Arabidopsis SRK2C/SnRK2.6 and wheat SnRK2.8 overexpressors were not changed by PtSnRK2.7 overexpression. Furthermore, $P t S n R K 2.7$ overexpression widely and largely influenced the transcriptome in response to salt stress; genes related to transport activity, including anion transport-related genes, were characteristically upregulated, and a variety of metabolic genes were specifically downregulated. We also found that the salt stress response genes were greatly upregulated in the PtSnRK2.7 overexpressor. Taken together, poplar subclass 2 PtSnRK2 genes can modulate salt stress tolerance in Arabidopsis, through the activation of cellular signaling pathways in a different manner from that by herbal subclass 2 SnRK2 genes.

Keywords: SnRK2, overexpression, salt stress, transport, metabolism, salt tolerance, poplar

\section{INTRODUCTION}

Plants face various environmental stresses including drought, high salinity, and extreme temperatures. Such adverse circumstances can often lead to severe agricultural and industrial losses, so it is important to understand the molecular and physiological mechanisms that plants use to cope with abiotic stresses for further stable production of crops and biomass feedstock.

Abbreviations: SnRK2, SNF1-related protein kinases 2; ABA, abscisic acid. 
Many studies have indicated that regulatory factors of protein phosphorylation play essential roles in response to environmental stimuli (Sopory and Munshi, 1998; Umezawa et al., 2013). One of the well-characterized protein kinases involved in stress responses is the group of sucrose nonfermenting 1 (SNF1)-related protein kinases (SnRKs; Halford and Hey, 2009). SnRKs are grouped into three subfamilies, SnRK1, SnRK2, and SnRK3 (Halford and Hey, 2009), and recent studies have indicated pivotal roles of plant-specific subgroups of SnRK2 and SnRK3 in the link between abiotic stress and abscisic acid (ABA) signaling to regulate metabolic pathways (Hrabak et al., 2003; Halford and Hey, 2009). Increasing evidence shows that SnRK2 proteins function as positive regulators of ABA signaling for stress responses, as well as development, in plants (Umezawa et al., 2013). In Arabidopsis thaliana (Arabidopsis) and rice, the SnRK2 family includes 10 members, such as SRK2ASRK2J or SnRK2.1-2.10 in Arabidopsis and SAPK1-10 in rice (Yoshida et al., 2002; Hrabak et al., 2003; Kobayashi et al., 2004), and they are further classified into three subclasses based on their domain structures (Kobayashi et al., 2004). Most SnRK2 proteins are activated by abiotic stresses, while the members of subclasses 2 and 3 are also activated by ABA (Boudsocq et al., 2004, 2007; Kobayashi et al., 2004). In the current model, ABA-induced activation is largely explained by the interaction between SnRK2s and protein phosphatase type $2 \mathrm{C}$ (PP2C) proteins in the ABA signaling pathway (Leung et al., 1994, 1997; Meyer et al., 1994; Saez et al., 2004; Nishimura et al., 2007; Umezawa et al., 2009; Cutler et al., 2010; $\mathrm{Ng}$ et al., 2014). In the absence of ABA, group A PP2Cs physically bind to SnRK2s to dephosphorylate SnRK2s, resulting in the inhibition of ABA signal transduction, while in the presence of ABA, SnRK2 will be released from such inhibitory regulation by $\mathrm{PP} 2 \mathrm{C}$, because the soluble $\mathrm{ABA}$ receptor PYR/PYL/RCAR inhibits PP2C activity (Umezawa et al., 2009; Vlad et al., 2009).

In Arabidopsis, detailed analyses of subclass 2 (SRK2F/SnRK2.7 and SRK2C/SnRK2.8) and subclass 3 (SRK2D/SnRK2.2, SRK2I/SnRK2.3, and SRK2E/SnRK2.6) have revealed their redundant functions in ABA signaling for abiotic stress responses and developmental controls (Yoshida et al., 2002; Fujii et al., 2007; Fujii and Zhu, 2009; Fujita et al., 2009; Nakashima et al., 2009; Mizoguchi et al., 2010). Importantly, overexpression of $S n R K 2$ genes resulted in enhanced abiotic stress tolerance in Arabidopsis (AtSRK2C/SnRK2.8, Umezawa et al., 2004; TaSnRK2.3, TaSnRK2.4, TaSnRK2.7, and TaSnRK2.8, Mao et al., 2010; Zhang et al., 2010, 2011; Tian et al., 2013) and in rice (SAPK4; Diédhiou et al., 2008). Overexpression of $\operatorname{SnRK2}$ genes in Arabidopsis induced the upregulation of several important stress responsive genes, including RD29A and DREB1A/CBF3, and ABA biosynthetic genes, such as ABA1, under normal conditions (Umezawa et al., 2004; Zhang et al., 2011), suggesting that early and quick stress responses supported by the expression of such key genes may enhance stress tolerance in Arabidopsis. The rice SAPK4 overexpressor showed increased salt tolerance, and major aspects of its tolerance were explained by changes in the expression of genes related to ion homeostasis and oxidative stress responses (Diédhiou et al., 2008). In the cases of SnRK2 overexpressors, the results clearly indicated that SnRK2 can function in abiotic stress responses in plant cells, through the modulation of stress response-related gene expression.

Comparative genomics studies have demonstrated that the core components of ABA signaling, PYR/PYL/RCAR, SnRK2, and $\mathrm{PP} 2 \mathrm{C}$, are well-conserved in land plant species (Umezawa et al., 2010), suggesting the evolutionary conservation of a molecular system involving these proteins in land plants. Indeed, the conserved molecular characteristics of SnRK2, such as transcriptional induction by abiotic stresses and activation by stress and/or ABA, have been reported for SnRK2 genes not only in Arabidopsis and rice, but also in other crop plants: maize (Huai et al., 2008; Vilela et al., 2012) and wheat (Holappa and Walker-Simmons, 1995; Gómez-Cadenas et al., 1999; Mao et al., 2010; Zhang et al., 2010, 2011; Tian et al., 2013). Additionally, we recently confirmed the physical interaction between SnRK2 and PP2C in a model tree Populus trichocarpa (poplar), and proposed the possibility that a similar molecular module containing SnRK2 and $\mathrm{PP} 2 \mathrm{C}$ is involved in the ABA signaling pathway in trees (Song et al., 2015). However, information about the molecular functions of the poplar SnRK2 proteins is still limited. Recently, we evaluated the transcriptional regulation of $P t S n R K 2$ genes and found that some of them are upregulated by abiotic stresses in organ-specific manners, suggesting the involvement of PtSnRK2 in ABA-dependent and/or ABA-independent regulation of stress responses ( $\mathrm{Yu}$ et al., unpublished data.).

In this study, to obtain further clues as to the molecular functions of PtSnRK2 proteins, we heterologously overexpressed the PtSnRK2 genes in Arabidopsis. Our data indicated that poplar subclass II PtSnRK2 genes can enhance the salt stress tolerance of Arabidopsis, and that poplar PtSnRK2 overexpression would activate cellular signaling and stress response pathways in Arabidopsis in a different manner than that by the herbal subclass II SnRK2 genes.

\section{MATERIALS AND METHODS}

\section{Plant Materials and Growth Conditions}

Young shoots of black cottonwood, P. trichocarpa Torr. \& A. Gray (poplar), grown in 15-cm-high plant pots, were used for the cloning of PtSnRK2 cDNA. For the overexpression analysis, A. thaliana (Arabidopsis) plants (Columbia strain) were used. The growth conditions were described in Ohtani et al. (2011) for poplar and in Ohtani et al. (2013) for Arabidopsis.

\section{Plasmid Construction and Transformation}

The coding sequences of PtSnRK2 genes were cloned into the Gateway entry vector pENTR/D-TOPO or pCR8/GW/TOPO (Invitrogen), as described by Song et al. (2015), and were transferred to the destination vector pH35GS (Kubo et al., 2005) by the LR reaction using LR clonase II (Invitrogen). In the resulting plasmids, the PtSnRK2 cDNA was expressed under the control of the cauliflower mosaic virus (CaMV) 35S promoter. The plasmids were electroporated into the Agrobacterium tumefaciens strain GV3101 (pMP90). A simplified version of the floral dip method was used for the transformation of Arabidopsis plants (Clough and Bent, 1998). 


\section{Establishment of Transgenic Lines}

For the screening of transgenic lines carrying the empty vector (vector controls) and 35S::PtSnRK2 (PtSnRK2 overexpressors), $\mathrm{T}_{1}$ seedlings were grown in germination Murashige and Skoog (MS) medium containing $50 \mu \mathrm{g} \mathrm{mL}^{-1}$ hygromycin for 2 weeks, and the positive plants were transferred to soil for further growth. Although we failed to generate overexpressors of PtSnRK2.2, $P t S n R K 2.6$, and PtSnRK2.8, more than 16 independent $\mathrm{T}_{2}$ lines were established for every other PtSnRK2 genes.

To evaluate the expression levels of the introduced PtSnRK2, total RNAs were prepared from 7-day-old seedlings of the wild type, vector controls, and PtSnRK2 overexpressors at the $\mathrm{T}_{2}$ generation using the RNeasy Mini kit (Qiagen). The first-strand cDNAs were synthesized using SuperScript III (Invitrogen) and subjected to RT-PCR analysis. The RT reaction was performed on a $20-\mu \mathrm{L}$ scale, with $1 \mu \mathrm{L}$ first-strand cDNA as a template for PCR, along with $0.5 \mu \mathrm{M}$ of each gene-specific primer (see Table S2) and Ex Taq polymerase (TaKaRa). The PCR conditions were as follows: for the internal control gene $U b p 10,95^{\circ} \mathrm{C}$ for $5 \mathrm{~min}$, followed by 35 cycles of $94^{\circ} \mathrm{C}$ for $20 \mathrm{~s}, 60^{\circ} \mathrm{C}$ for $30 \mathrm{~s}$, and $72^{\circ} \mathrm{C}$ for $15 \mathrm{~s}$, followed by $72^{\circ} \mathrm{C}$ for $7 \mathrm{~min}$; for PtSnRK2 genes (target genes), $95^{\circ} \mathrm{C}$ for $5 \mathrm{~min}$, followed by 35 cycles of $94^{\circ} \mathrm{C}$ for $20 \mathrm{~s}, 60^{\circ} \mathrm{C}$ for $30 \mathrm{~s}$, and $72^{\circ} \mathrm{C}$ for $1 \mathrm{~min} 15 \mathrm{~s}$, and followed by $72^{\circ} \mathrm{C}$ for $7 \mathrm{~min}$. The amplified PCR products were electrophoresed in 3 and $2 \%(\mathrm{w} / \mathrm{v})$ agarose gels for the Ubp10 and PtSnRK2 genes, respectively, and gel images were analyzed using the AE-9020 Eshot II (ATTO; Figure S1). Information about the primer sets is provided in Table S11.

\section{Salt Stress Treatment}

It is generally known that the expression level of $35 \mathrm{~S}$ promoterdriven genes would be decreased in $\mathrm{T}_{3}$ homozygous lines, so we decided to use $\mathrm{T}_{2}$ lines that were confirmed to show high expression levels of inserted PtSnRK genes as described above, for the salt stress treatment. Wild-type and transgenic $\mathrm{T}_{2}$ plants were grown at $22^{\circ} \mathrm{C}$ under $\mathrm{LD}$ conditions ( $16 \mathrm{~h}$ light/ $8 \mathrm{~h}$ dark) on $1 / 2$ strength MS medium, after incubation at $4^{\circ} \mathrm{C}$ for 3 days. Then, 7-day-old plants were transferred to $1 / 2$-MS medium plates with or without $\mathrm{NaCl}$. For the survival rates and chlorophyll contents, $200 \mathrm{mM} \mathrm{NaCl}$ was included in the medium, and for the root growth phenotype, $100 \mathrm{mM} \mathrm{NaCl}$ was used. After an additional 4day incubation, the numbers of surviving seedlings were counted to obtain survival rates $(n=20)$, and chlorophyll contents were measured as described below $(n=10)$. The primary root length was measured before and after a 5-day incubation on the $\mathrm{NaCl}$ plate, to calculate primary root elongation during salt stress treatment $(n=10)$. The treatments were repeated three times for survival rates and root length and six times for chlorophyll contents.

\section{Chlorophyll Quantitation}

Ten seedlings treated with $200 \mathrm{mM} \mathrm{NaCl}$ for 4 days were sampled in $3 \mathrm{~mL}$ ( $N, N$-dimethylformamide, DMF). After incubation in DMF overnight at $4^{\circ} \mathrm{C}$ in the dark, $\mathrm{A}_{646.8}, \mathrm{~A}_{663.8}$, and $\mathrm{A}_{750}$ were measured using the iMark Microplate Absorbance Reader (Bio-Rad). The chlorophyll quantitation was calculated by the formula: $\left[8.05 \times\left(\mathrm{A}_{663.8}-\mathrm{A}_{750}\right)+19.43 \times\left(\mathrm{A}_{646.8}-\mathrm{A}_{750}\right)(\mu \mathrm{M}) /\right.$ the quantity of seedlings $(\mathrm{mg})](\mu \mathrm{M} / \mathrm{mg})$ (Porra et al., 1989).

\section{In silico Prediction of the Three-Dimensional Structures of PtSnRK2 Proteins}

The amino acid sequences of PtSnRK2.5, PtSnRK2.7, and PtSnRK2.9 were submitted to the web-based SWISS-MODEL service (http://swissmodel.expasy.org/workspace/; Arnold et al., 2006), to build protein structure homology models using information on the crystal structure of the recombinant AtSnRK2.6 protein as a template (Ng et al., 2011).

\section{Microarray Analysis}

Seedlings of the wild-type and PtSnRK2.7 overexpressor line 20 treated with or without $200 \mathrm{mM} \mathrm{NaCl}$ for 2 days were sampled for total RNA extraction. Microarray analysis was performed using ATH1 GeneChips (Affymetrix) according to the manufacturer's instructions on three independent biological replicates. Subsequent procedures of quality control, statistical analysis, and filtering were carried out using GeneSpring GX software (ver. 13.1; Agilent Technologies). Then, $p$-values were calculated for each probe using Welch's $t$-test $(n=3)$ for differences between the treated seedlings and the control seedlings, as well as between the wild-type and PtSnRK2.7 overexpressor incubated without salt treatment. We used the Benjamin-Hochberg FDR method to control for false positives. A $p$-value cut-off of 0.01 was used to select genes whose expression changed with salt treatment. Fold-change values were also computed using GeneSpring GX, and we targeted those probes in which the change was upregulated or downregulated by more than 3-fold. Microarray data presented in this study were submitted to NCBI GEO (www.ncbi.nlm.nih.gov/geo/) and can be retrieved via accession number GSE79997.

\section{Gene Ontology (GO) Term Analysis}

Gene ontology (GO) term analysis was performed using the PANTHER classification system (Overrepresentation Test, release 20150430; Mi et al., 2013).

\section{Quantitative RT-PCR Analysis}

To evaluate the enhanced upregulation of salt stress response genes in PtSnRK2.7 overexpressors, quantitative RT-PCR analysis was performed. Total RNAs were isolated from the seedlings treated with or without $200 \mathrm{mM} \mathrm{NaCl}$ for 2 days using Plant RNA Isolation Reagent (Invitrogen) and then purified using the RNeasy Mini Kit (QIAGEN). The first-strand cDNAs were synthesized as described above, and aliquots of the cDNA solution $(0.5 \mu \mathrm{L}$ for each gene) were used as templates for subsequent PCR amplification. The quantitative PCR analysis was performed using the LightCycler 480 System II (Roche) and LightCycler 480 SYBR Green I Master reagents (Roche). As an internal control, the $U b c 9$ gene was used. Information on the primer sets is provided in Table S11. 


\section{RESULTS AND DISCUSSION}

\section{Overexpression of PtSnRK2.5 and PtSnRK2.7 Improved Salt Stress Tolerance in Arabidopsis}

In the genome of $P$. trichocarpa (poplar), $12 \mathrm{PtSnRK} 2$ genes have been identified (Song et al., 2015). For molecular functional analysis of PtSnRK2, we generated transgenic plants of A. thaliana (Arabidopsis) carrying the chimeric gene $35 S:: P t S n R K 2$, in which the cDNA regions of PtSnRK2 genes were regulated by the CaMV $35 \mathrm{~S}$ promoter sequence, to overexpress poplar PtSnRK2 genes in Arabidopsis. Unfortunately we could not obtain transgenic plants for PtSnRK2.2, PtSnRK2.6, and PtSnRK2.8 overexpression; however, overexpressors of the other nine PtSnRK2 genes were established successfully. It has been reported that overexpression of SnRK2 genes enhances abiotic stress tolerance (Umezawa et al., 2004; Diédhiou et al., 2008; Mao et al., 2010; Zhang et al., 2010, 2011; Tian et al., 2013). Thus, we examined salt stress tolerance in the PtSnRK2 overexpressors.

First, 7-day-old seedlings from 16 independent $T_{2}$ lines for each PtSnRK2 overexpressor were transferred to medium containing $200 \mathrm{mM} \mathrm{NaCl}$, and incubated for 4 days. The prr911 prr7-10 prr5-10 triple mutant (d975) plant, which showed high salinity tolerance because of high expression of salt stress response genes (Nakamichi et al., 2009), was used as a positive control (Figure S2). The survival rates of seedlings after salt treatment demonstrated that the overexpressors of PtSnRK2.5 and PtSnRK2.7 showed relatively high survival rates among the PtSnRK2 overexpressors. These two genes encode the subclass 2 PtSnRK2 proteins (Song et al., 2015). We further performed a detailed analysis of salt stress tolerance in the PtSnRK2.5 and PtSnRK2.7 overexpressors, using the wild-type and vector control plants as negative controls (Figures 2, 3). For the PtSnRK2.5 and PtSnRK2.7 overexpressors, three independent lines were selected based on the expression levels of the introduced PtSnRK2 genes (Figure S1). In the case of the negative controls, the seedling survival rates after $200 \mathrm{mM}$ $\mathrm{NaCl}$ treatment were less than 20\%, and the living seedlings exhibited yellowed leaves (Figure 2). In contrast, the PtSnRK2.5 and PtSnRK2.7 transgenic seedlings showed significantly higher survival rates $(\sim 55 \%)$ than those of the controls (Figure 2$)$. All three independent PtSnRK2.7 lines showed increased survival rates, whereas the $P t S n R K 2.5$ line 6 did not show a significantly enhanced survival rate (Figure 2B).

Next, the chlorophyll contents of 10 seedlings treated with salt stress were analyzed. The salt treatment greatly decreased chlorophyll contents in all plants; however, the chlorophyll contents of PtSnRK2.5 lines 16 and 20, and all of the PtSnRK2.7 lines were significantly higher than those of the wild-type and vector control (Figure 2C), in accordance with the survival rates. These results suggested that salt stress tolerance was more stable in the PtSnRK2.7 overexpressors. Moreover, it was notable that although the chlorophyll contents in the absence of salt stress were at almost the same levels among the wild-type, vector control, and PtSnRK2.7 lines, but the PtSnRK2.5 lines 16 and 20 showed lower amounts of chlorophyll under normal growth conditions (Figure 2C). Thus, PtSnRK2.5 overexpression may have affected chlorophyll biosynthesis continuously.

In addition to the increased survival rates and chlorophyll contents, the living seedlings of the PtSnRK2.5 and PtSnRK2.7 transgenic lines seemed to be larger than those of the negative controls under salt stress conditions (Figure 2A). To clarify the effects of PtSnRK2 overexpression on seedling growth, we checked the growth of primary roots after salt treatment. Because the $200 \mathrm{mM} \mathrm{NaCl}$ treatment almost completely inhibited primary root elongation in both the overexpressors and negative controls, we used $100 \mathrm{mM} \mathrm{NaCl}$ conditions to observe primary root elongation. Our data demonstrated no difference in primary root elongation in the mock-treated seedlings (Figure 3), indicating that these two PtSnRK2 genes are not involved in root elongation regulation, unlike the Arabidopsis subclass II AtSRK2C/SnRK2.8 gene, overexpression of which was reported to enhance seedling root growth (Shin et al., 2007). However, after a 5-day incubation with $100 \mathrm{mM} \mathrm{NaCl}$, the primary roots of PtSnRK2.5 line 20 and all of the PtSnRK2.7 lines elongated significantly more than did those of the negative controls; the negative controls showed elongation of less than $20 \mathrm{~mm}$, whereas the elongated lengths of PtSnRK2.5 line 20 and all of the PtSnRK2.7 lines were $\sim 25 \mathrm{~mm}$ ( $p$ $<0.01, t$-test). These observations indicated that overexpression of PtSnRK2.7, and possibly also PtSnRK2.5, can suppress the inhibition of root elongation by high salinity stress.

Our data on the PtSnRK2.5 and PtSnRK2.7 transgenic lines demonstrated different phenotypic characteristics between the PtSnRK2.5 and PtSnRK2.7 lines. Thus, it was suspected that the molecular basis for the enhancement of salt stress tolerance would be different between the PtSnRK2.5 and PtSnRK2.7 transgenic lines. In particular, the PtSnRK2.5 lines showed unstable salt stress tolerance (Figures 2, 3) and defects in chlorophyll content even under normal growth condition (Figure 2C). The subclass $2 \mathrm{PtSnRK} 2$ proteins have highly similar amino acid sequences (Figure 1A), and the amino acid sequences of PtSnRK2.5 (unstable, but significantly enhanced salinity tolerance), PtSnRK2.7 (stable and high tolerance), and PtSnRK2.9 (no obviously increased tolerance) did not show major differences between them (Figure 1A). In silico modeling of the three-dimensional structures of these proteins, based on the crystal structure of AtSnRK2.6 (Ng et al., 2011), revealed that some of the differences in amino acid sequences among these PtSnRK2 proteins could correspond to the molecular surface regions of these proteins (Figure 1B), possibly leading to changes in the molecular activities of the SnRK2 proteins. The different SnRK2 protein activities due to subtle substitutions of amino acids could result in the different strengths of salt stress tolerance among the overexpressors.

\section{PtSnRK2.7 Overexpression Widely and Largely Influenced the Transcriptome in Response to Salt Stress}

We next performed transcriptomic analyses of the wild-type and overexpressors during salt stress treatment. Based on the data described above (Figures 2, 3), we selected PtSnRK2.7 line 20 , which showed stable and strong salt stress tolerance 


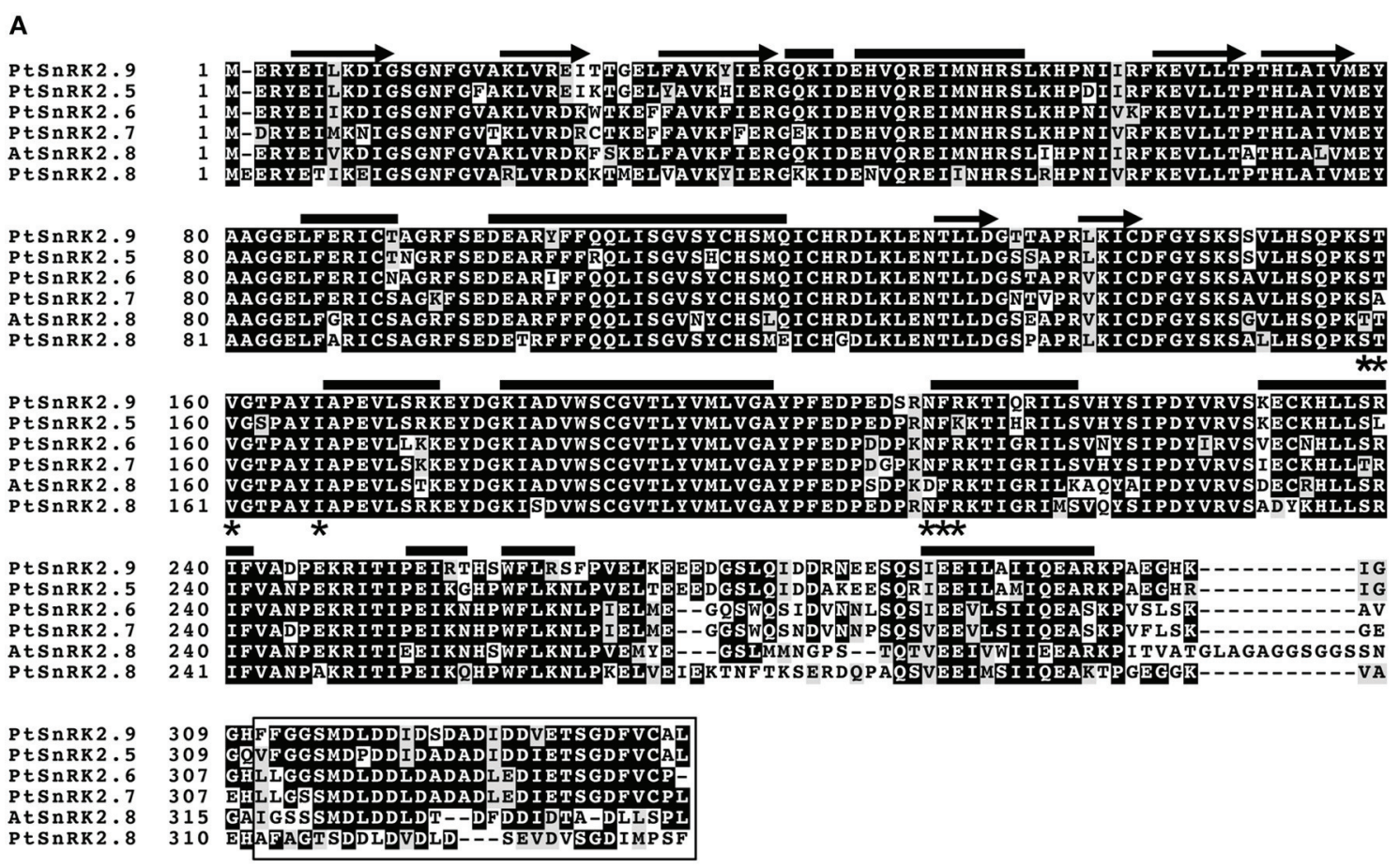

B

PtSnRK2.7

(stable tolerance

in its overexpressor)

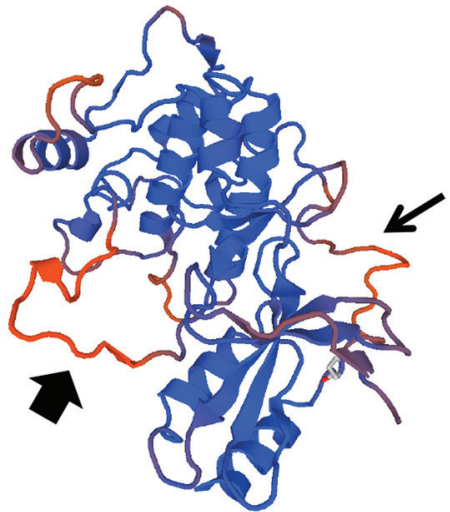

PtSnRK2.5

(unstable tolerance

in its overexpressor)

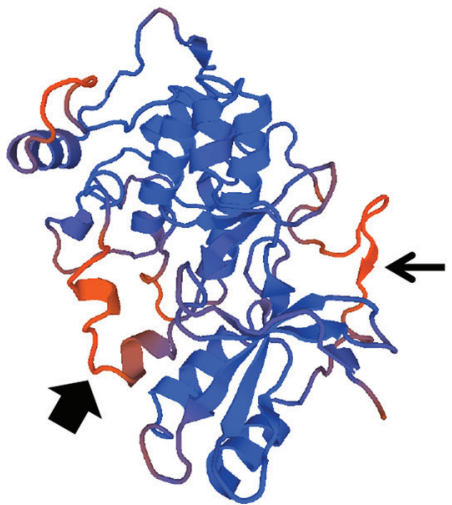

PtSnRK2.9

(no tolerance

in its overexpressor)

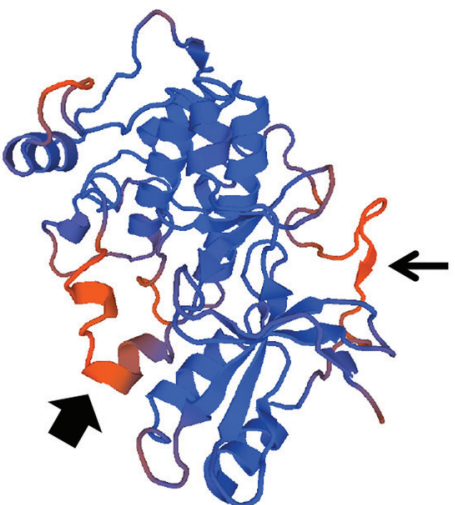

FIGURE 1 | SnRK2 proteins of Populus trichocarpa (poplar, Pt), and Arabidopsis thaliana (Arabidopsis, At). (A) Amino acid sequence alignment of subclass $2 \mathrm{PtSnRK} 2$ proteins with AtSnRK2.8. The residues putatively corresponding to the $\alpha$-helix, $\beta$-sheet, PP2C-interaction residues, and the entire ABA box are marked by bars, arrows, asterisks, and the box, respectively. (B) In silico modeling of the three-dimensional structures of PtSnRK2.5, PtSnRK2.7, and PtSnRK2.9 proteins. Homology modeling was performed based on the crystal structure of AtSnRK2.6 using the web-based SWISS-MODEL service (http://swissmodel.expasy.org/ workspace/; Arnold et al., 2006). Thick and narrow arrows indicate regions presumed to differ in their three-dimensional architecture among the PtSnRK2 proteins because of differences in amino acid sequences.

among the transgenic lines, for the gene chip analysis. Then, 7-day-old seedlings of the wild-type and PtSnRK2.7 line 20 were treated with or without $200 \mathrm{mM} \mathrm{NaCl}$ for 2 days and then sampled to extract total RNA. The extracted total RNA samples were subjected to microarray analysis using Affymetrix ATH1 GeneChips (Figure 4A). First, we compared transcriptomic data between the wild-type and PtSnRK2.7 overexpressor, which were mock-treated, to examine the effects of PtSnRK2.7 overexpression on gene expression under normal growth conditions. Thirty and 79 genes were shown to be upregulated and downregulated in the PtSnRK2.7 overexpressor, respectively ( $\mathrm{FC}>2, p<0.05$; Table $\mathrm{S} 1$ ). It has been reported 
A

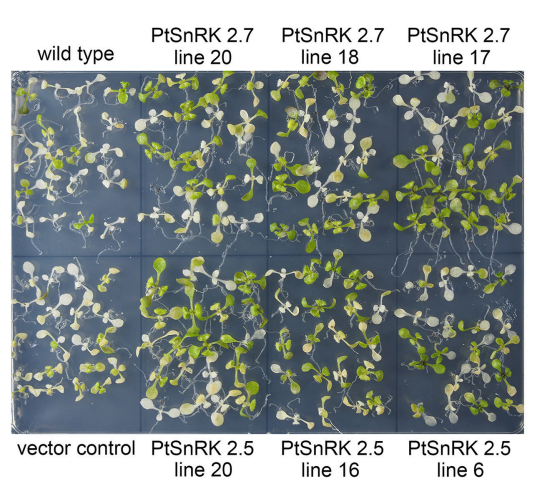

B

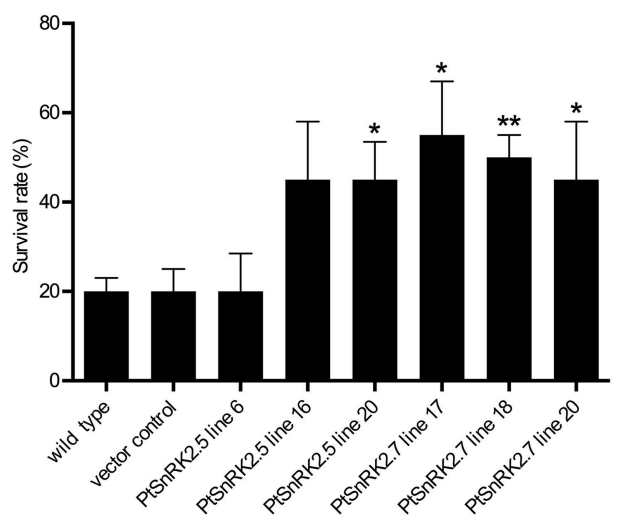

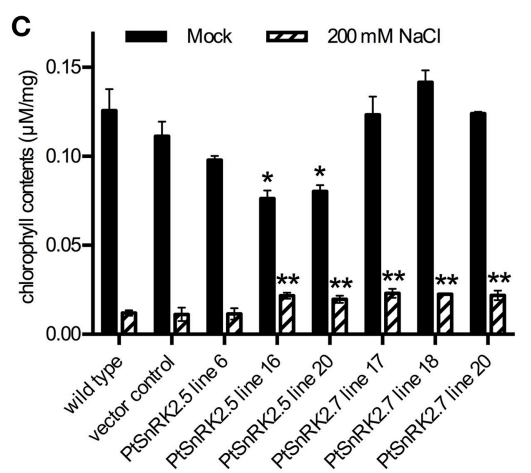

FIGURE 2 | High salinity tolerance phenotype of transgenic Arabidopsis overexpressing PtSnRK2.5 and PtSnRK7. (A) Seedlings of the wild-type, vector control, and overexpressors of PtSnRK2.5 and PtSnRK2.7 treated with $200 \mathrm{mM} \mathrm{NaCl}$ for 4 days. (B) Survival rates determined by observations after a 4-day salt stress treatment. The green seedlings were counted as living seedlings, and the percentages of live seedlings were calculated using 20 seedlings for each line. (C) Chlorophyll contents determined from 20 seedlings after a 4-day salt stress treatment. Results are means \pm SE $(n=3)$. Asterisks indicate statistically significant differences between transgenic and wild-type plants (Student's $t$-test; ${ }^{*} p<0.05$; ${ }^{* *} p<0.01$ ).

that in AtSRK2C/SnRK2.6 and TaSnRK2.8 overexpressors, stress response-related genes including RD29A and DREB1A/CBF3, and ABA biosynthetic genes are upregulated continuously (Umezawa et al., 2004; Zhang et al., 2011). However, these genes were not changed by PtSnRK2.7 overexpression (Table S1). The gene ontology (GO) term analysis revealed that stress-related metabolic genes, including lipid metabolism and flavonoid metabolism, were significantly downregulated in the PtSnRK2.7 overexpressor (Table 1). These results suggest that PtSnRK2.7 overexpression could continuously affect specific ranges of gene expression regulation, which do not overlap with the primary targets of AtSRK2C/SnRK2.6 and TaSnRK2.8 in Arabidopsis.

To examine the impact of PtSnRK2.7 overexpression on the salt stress response, the genes upregulated or downregulated by salt treatment were compared between the wild-type and PtSnRK2.7 overexpressor. In the wild-type, 337 and 120 genes were upregulated and downregulated by salt treatment, respectively (FC $>3, p<0.01$; Figure $4 \mathbf{B}$ and Tables S2, S3). Notably, the PtSnRK2.7 overexpressor showed greater numbers of genes whose expression was changed by salt treatment compared with the wild-type; 631 and 698 genes were upregulated and downregulated in the PtSnRK2.7 overexpressor, respectively ( $\mathrm{FC}>3, p<0.01$; Figure $4 \mathrm{~B}$ and Tables S4, S5), suggesting that PtSnRK2.7 overexpression affected the expression of a wide range of genes during salt stress responses in Arabidopsis. The GO analysis indicated that the genes functioning in stress responses, including the signaling pathway of stress-related phytohormones (ABA, ethylene, jasmonic acid, and salicylic acid) and in signal transduction by protein phosphorylation, were commonly upregulated between the wild-type and PtSnRK2.7 overexpressor (Figure 4B and Tables S6-S8). Additionally, developmental process-related genes were commonly downregulated in the wild-type and PtSnRK2.7 overexpressor (Figure 4B and Table S10).

We also found that some GO terms were enriched in the upregulated genes in genotype-dependent manners, such as "respiratory burst" and "ethylene biosynthetic process" found only in the wild-type (Figure 4B and Table S6) and "purine nucleoside transmembrane transport," "amino acid transport," "anion transmembrane transport," and "nucleic acid metabolic process" found only in the PtSnRK2.7 overexpressor (Figure 4B and Table S7). The overrepresentation of the GO terms related to "anion transmembrane transport" in the PtSnRK2.7 overexpressor would suggest the enhancement of ion 




\section{B}

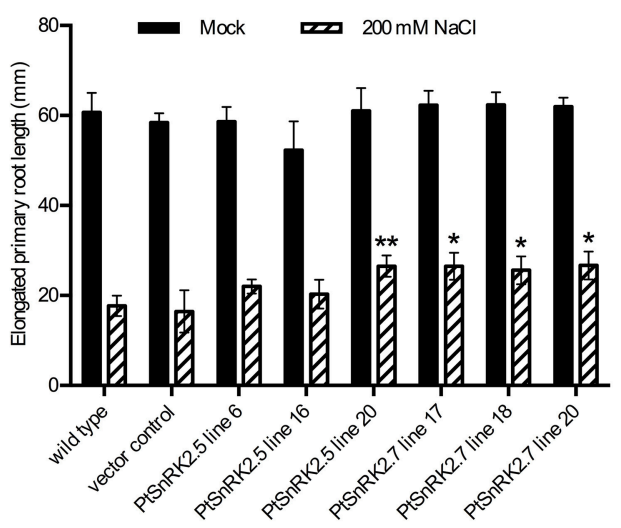

FIGURE 3 | Primary root elongation of transgenic Arabidopsis overexpressing PtSnRK2.5 and PtSnRK2.7 during salt treatment. (A) Seedlings of the wild-type, vector control, and overexpressors of PtSnRK2.5 and PtSnRK2.7 treated with $100 \mathrm{mM} \mathrm{NaCl}$ for 5 days. The positions of the edges of the root tips before salt treatment are indicated by the white dotted lines. Bar $=20 \mathrm{~mm}$. (B) Increase in root length after salt stress treatment. Results are means $\pm S E(n=15)$. Asterisks indicate statistically significant differences between transgenic plants and the wild-type (Student's $t$-test; ${ }^{*} p<0.05 ;{ }^{* *} p<0.01$ ).

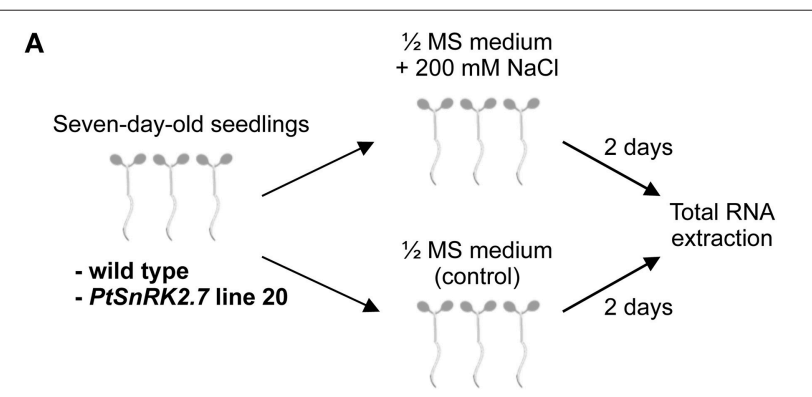

B Upregulated by salt

\section{Downregulated by salt}

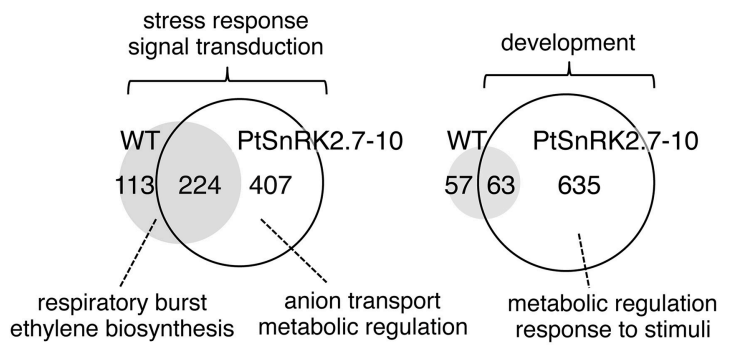

FIGURE 4 | Microarray analysis of the wild-type and PtSnRK2.7 overexpressor line 20. (A) Overview of sample preparation for microarray analysis. (B) Venn diagram of upregulated and downregulated genes after salt stress treatment in the wild-type and PtSnRK2.7 overexpressor. homeostasis activity as a result of PtSnRK2.7 overexpression, possibly leading to higher salt stress tolerance. Our results also showed wild-type-specific enrichment of the term "ethylene biosynthetic process" in upregulated genes (Figure 4B and Table S6). It has been reported that crosstalk between ABA and ethylene is a critical factor in determining salt stress tolerance in Arabidopsis (Dong et al., 2011); thus, PtSnRK2.7 overexpression may influence the phytohormonal modulating system of abiotic stress responses. Moreover, in the PtSnRK2.7 overexpressor, more than 5-fold more genes were downregulated by salt treatment than those in the wild type. These downregulated genes are involved in a wide range of molecular functions, including metabolic regulation and responses to stimuli (Figure $4 \mathrm{~B}$ and Table 59), indicating that the reason for the high salt stress tolerance in the PtSnRK2.7 overexpressor may be, at least partially, attributed to its significant impact on metabolic regulation.

Comparison of transcriptomic data also suggested that the altered expression levels of upregulated genes were greater in the PtSnRK2.7 overexpressor than in the wild-type (Tables S2, S4). To confirm this, we selected six genes from the functional categories of stress response (COR15A, Artus et al., 1996), phytohormonal signaling (GASA3, Herzog et al., 1995), cell wall-related proteins (AT1G52690 and AtCWINV5, Sherson et al., 2003), and lipid metabolism (AT4G33550), based on the transcriptomic data, and subjected them to quantitative 


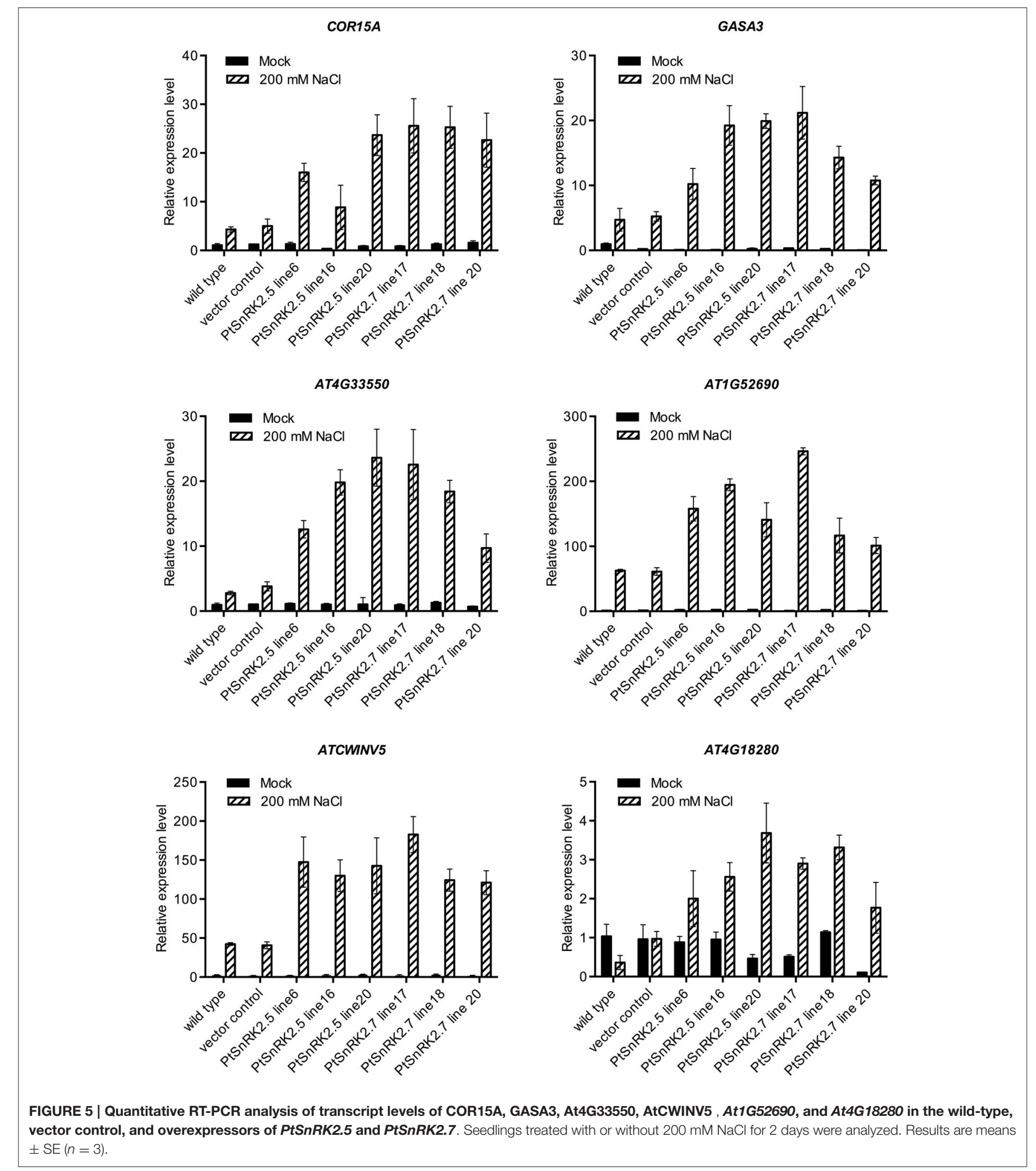

RT-PCR analysis. AT4G18280, which was upregulated only in the PtSnRK2.7 overexpressor, was also tested to evaluate the consistency of the results between the gene chip analysis and quantitative RT-PCR analysis. Indeed, AT4G18280 expression was not induced by salt stress in the wild-type or vector control, whereas it was highly upregulated in the PtSnRK2.7 line 20 in accordance to the results of gene chip analysis (Figure 5 and Table S4). The results showed that the tested genes were actively 
TABLE 1 | GO term analysis of the differentially expressed genes between the wild-type and PtSnRK2.7 overexpressor grown in the absence of salt stress treatment.

\begin{tabular}{|c|c|c|}
\hline GO biological process complete & $\begin{array}{l}\text { Fold } \\
\text { Enrichment }\end{array}$ & $p$-value \\
\hline $\begin{array}{l}\text { Anthocyanin-containing compound } \\
\text { biosynthetic process }\end{array}$ & 44.44 & 4.52E-03 \\
\hline $\begin{array}{l}\text { Anthocyanin-containing compound } \\
\text { metabolic process }\end{array}$ & 40.00 & 7.29E-03 \\
\hline $\begin{array}{l}\text { Cellular response to phosphate } \\
\text { starvation }\end{array}$ & 33.33 & 1.12E-09 \\
\hline Galactolipid biosynthetic process & 33.33 & 2.61E-07 \\
\hline Galactolipid metabolic process & 33.33 & 2.61E-07 \\
\hline Glycolipid biosynthetic process & 28.57 & 1.06E-06 \\
\hline Flavonoid biosynthetic process & 26.09 & 2.69E-04 \\
\hline Flavonoid metabolic process & 24.00 & 4.11E-04 \\
\hline Liposaccharide metabolic process & 23.53 & 4.26E-06 \\
\hline Glycolipid metabolic process & 23.53 & 4.26E-06 \\
\hline $\begin{array}{l}\text { Membrane lipid biosynthetic } \\
\text { process }\end{array}$ & 22.22 & 6.69E-06 \\
\hline Response to UV-B & 20.83 & 9.49E-03 \\
\hline Cellular response to starvation & 18.18 & 6.92E-09 \\
\hline Cellular response to nutrient levels & 17.65 & 8.89E-09 \\
\hline Membrane lipid metabolic process & 17.39 & 4.15E-05 \\
\hline Response to starvation & 17.39 & $1.08 \mathrm{E}-08$ \\
\hline $\begin{array}{l}\text { Cellular response to extracellular } \\
\text { stimulus }\end{array}$ & 16.88 & $1.88 \mathrm{E}-09$ \\
\hline $\begin{array}{l}\text { Cellular response to external } \\
\text { stimulus }\end{array}$ & 16.67 & 2.26E-09 \\
\hline Response to nutrient levels & 16.67 & 1.82E-08 \\
\hline Response to extracellular stimulus & 16.05 & 3.73E-09 \\
\hline Pigment biosynthetic process & 13.73 & 1.65E-03 \\
\hline Pigment metabolic process & 10.94 & 7.65E-03 \\
\hline $\begin{array}{l}\text { Negative regulation of transcription, } \\
\text { DNA-templated }\end{array}$ & 8.43 & 3.85E-02 \\
\hline $\begin{array}{l}\text { Negative regulation of RNA } \\
\text { biosynthetic process }\end{array}$ & 8.43 & 3.94E-02 \\
\hline $\begin{array}{l}\text { Negative regulation of nucleic } \\
\text { acid-templated transcription }\end{array}$ & 8.43 & 3.94E-02 \\
\hline $\begin{array}{l}\text { Negative regulation of RNA } \\
\text { metabolic process }\end{array}$ & 8.43 & 4.03E-02 \\
\hline Cellular response to stress & 5.62 & 1.09E-04 \\
\hline Response to external stimulus & 3.65 & 2.57E-03 \\
\hline $\begin{array}{l}\text { Single-organism biosynthetic } \\
\text { process }\end{array}$ & 2.99 & 6.83E-03 \\
\hline
\end{tabular}

upregulated by salt treatment in the PtSnRK2.7 overexpressors compared with the wild-type and vector control (Figure 5). Our data also demonstrated that PtSnRK2.5 overexpression had similar effects on the expression of these genes. Thus, it is supposed that PtSnRK2.5 overexpression affected the transcriptome in a similar fashion to PtSnRK2.7 overexpression.

It has been reported that the overexpression of Arabidopsis AtSnRK2.8 enhanced the stress tolerance of Arabidopsis, probably through the continuous upregulation of key genes for stress responses, such as RD29A, COR15A, AtGolS3, DREB1A, and PKS18 (Umezawa et al., 2004). However, our results showed that overexpression of poplar SnRK2 genes did not constitutively induce these well-known stress responsive genes. Rather, the high salt stress tolerance of the PtSnRK2 overexpressors may be explained by changes in transcriptomic regulation for a wide range of metabolic regulatory genes (Figures 4, 5; Table 1 and Tables S1-S10). It is notable that the genes related to anion transport activity were upregulated in the PtSnRK2.7 overexpressor specifically, because similar effects on ion homeostasis-related genes were reported in the case of overexpression of rice SAPK4 (Diédhiou et al., 2008). Moreover, a comparative transcriptomic analysis using salt-tolerant and non-tolerant species of poplar indicated that prominent factors for high salt tolerance were not overexpression of the stress responsive pathway, but rather enhanced activities for osmotic adjustment, ion compartmentalization, and detoxification of reactive oxygen species in poplar (Chen and Polle, 2010). Thus, our results may reflect differences in the regulatory targets of SnRK2 proteins between AtSnRK2 and PtSnRK2, which could be related to diversified molecular strategies of stress adaption in each plant species, as suggested by Zhang et al. (2014). Future comparative analyses on the mode of actions of SnRK2 proteins derived from different plant species may provide important information on novel strategies to improve stress tolerance of crops and other useful plants.

\section{AUTHOR CONTRIBUTIONS}

XS designed and performed the experiments, and drafted the manuscript. MO designed the experiments, analyzed the data, and wrote the manuscript. $\mathrm{XY}$ and $\mathrm{CH}$ contributed to the data analysis. QZ and TD drafted the manuscript. All authors contributed to and approved the final manuscript.

\section{ACKNOWLEDGMENTS}

We thank Ms. Ayumi Ihara, Ms. Arika Takebayashi and Ms. Rokyo Hiroyama (RIKEN) for their technical assistance. This work was supported by the International Science \& Technology Cooperation Program of China (2014DFG32440), the National Science Foundation of China (No.31570650), the Priority Academic Program Development of Jiangsu Higher Education Institutions, the Program for Innovative Research Team in University of Educational Department and Jiangsu Province, China, and the Biomass Engineering Program Cooperation Division, RIKEN Center for Sustainable Resource Science, Japan.

\section{SUPPLEMENTARY MATERIAL}

The Supplementary Material for this article can be found online at: http://journal.frontiersin.org/article/10.3389/fpls.2016. 00612

Figure S1 | RT-PCR analysis of seedlings of the wild-type, vector control, and overexpressors of PtSnRK2.5 and PtSnRK2.7.

Figure S2 | Primary screening of transgenic Arabidopsis overexpressing PtSnRK2 genes for the salt tolerance. Seedlings of the d975 (positive control), vector control, and overexpressors of PtSnRK2 genes were treated with $200 \mathrm{mM}$ $\mathrm{NaCl}$, and checked their survival rates after 3,4 , and 5 days of $\mathrm{NaCl}$ treatment. A 
part of results for the transgenic lines of PtSnRK2.5 (A) and PtSnRK2.9 (B) treated with $200 \mathrm{mM} \mathrm{NaCl}$ for 4 days were shown.

Table S1 | Differentially expressed genes between the wild type and PtSnRK2.7 overexpressor (FC $>2, p<0.05$ ).

Table S2 | Upregulated 337 genes in the wild type by the salt treatment (FC>3, $p<0.01$ ).

Table S3 | Downregulated 120 genes in the wild type by the salt treatment (FC>3, $p<0.01$ ).

Table S4 | Upregulated 631 genes in the PtSnRK2.7 overexpressor by the salt treatment $(\mathrm{FC}>3, p<0.01)$.

Table S5 | Downregulated 698 genes in the PtSnRK2.7 overexpressor by the salt treatment $(\mathrm{FC}>3, p<0.01)$.

\section{REFERENCES}

Arnold, K., Bordoli, L., Kopp, J., and Schwede, T. (2006). The SWISSMODEL Workspace: a web-based environment for protein structure homology modelling. Bioinformatics 22, 195-201. doi: 10.1093/bioinformatics/bti770

Artus, N. N., Uemura, M., Steponkus, P. L., Gilmour, S. J., Lin, C., and Thomashow, M. F. (1996). Constitutive expression of the cold-regulated Arabidopsis thaliana COR15a gene affects both chloroplast and protoplast freezing tolerance. Proc. Natl. Acad. Sci. U.S.A. 93, 13404-13409. doi: 10.1073/pnas.93.23.13404

Boudsocq, M., Barbier-Brygoo, H., and Laurière, C. (2004). Identification of nine sucrose nonfermenting 1-related protein kinases 2 activated by hyperosmotic and saline stesses in Arabidopsis thaliana. J. Biol. Chem. 279, 41758-41766. doi: 10.1074/jbc.M405259200

Boudsocq, M., Droillard, M. J., Barbier-Brygoo, H., and Laurière, C. (2007). Different phosphorylation mechanisms are involved in the activation of sucrose non-fermenting 1 related protein kinases 2 by osmotic stresses and abscisic acid. Plant Mol. Biol. 63, 491-503. doi: 10.1007/s11103-006-9103-1

Chen, S., and Polle, A. (2010). Salinity tolerance of Populus. Plant Biol. 12, 317-333. doi: 10.1111/j.1438-8677.2009.00301.x

Clough, S. J., and Bent, A. F. (1998). Floral dip: a simplified method for Agrobacterium-mediated transformation of Arabidopsis thaliana. Plant J. 16, 735-743. doi: 10.1046/j.1365-313x.1998.00343.x

Cutler, S. R., Rodriguez, P. L., Finkelstein, R. R., and Abrams, S. R. (2010). Abscisic acid: emergence of a core signaling network. Annu. Rev. Plant Biol. 61, 651-679. doi: 10.1146/annurev-arplant-042809-112122

Diédhiou, C. J., Popova, O. V., Dietz, K. J., and Golldack, D. (2008). The SNF1type serine-threonine protein kinase SAPK4 regulates stress-responsive gene expression in rice. BMC Plant Biol. 8:49. doi: 10.1186/1471-2229-8-49

Dong, H., Zhen, Z., Peng, J., Chang, L., Gong, Q., and Wang, N. N. (2011). Loss of ACS7 confers abiotic stress tolerance by modulating ABA sensitivity and accumulation in Arabidopsis. J. Exp. Bot. 62, 4875-4887. doi: 10.1093/jxb/ err143

Fujii, H., Verslues, P. E., and Zhu, J. K. (2007). Identification of two protein kinases required for abscisic acid regulation of seed germination, root growth, and gene expression in Arabidopsis. Plant Cell 19, 485-494. doi: 10.1105/tpc.106.048538

Fujii, H., and Zhu, J. K. (2009). Arabidopsis mutant deficient in 3 abscisic acid-activated protein kinases reveals critical roles in growth, reproduction, and stress. Proc. Natl. Acad. Sci. U.S.A. 106, 8380-8385. doi: 10.1073/pnas.0903144106

Fujita, Y., Nakashima, K., Yoshida, T., Katagiri, T., Kidokoro, S., Kanamori, N., et al. (2009). Three SnRK2 protein kinases are the main positive regulators of abscisic acid signaling in response to water stress in Arabidopsis. Plant Cell Physiol. 50, 2123-2132. doi: 10.1093/pcp/pcp147

Gómez-Cadenas, A., Verhey, S. D., Holappa, L. D., Shen, Q., Ho, T. -H. D., and Walker-Simmons, M. K. (1999). An abscisic acid-induced protein kinase, PKABA1, mediates abscisic acid-suppressed gene expression in barley aleurone layers. Proc. Natl. Acad. Sci. U.S.A. 96, 1767-1772. doi: 10.1073/pnas.96.4.1767

Halford, N. G., and Hey, S. J. (2009). Snf1-related protein kinases (SnRKs) act within an intricate network that links metabolic and stress signalling in plants. Biochem. J. 419, 247-259. doi: 10.1042/BJ20082408
Table S6 | GO term analysis on wild-type-specific upregulated genes by salt treatment.

Table S7 | GO term analysis on PrSnRK2.7 overexpressor-specific upregulated genes by salt treatment.

Table S8 | GO term analysis on commonly upregulated genes between the wild type and PtSnRK2.7 overexpressor by salt treatment.

Table S9 | GO term analysis on PrSnRK2.7 overexpressor-specific downregulated genes by salt treatment.

Table S10 | GO term analysis on commonly downregulated genes between the wild type and PtSnRK2.7 overexpressor by salt treatment.

Table S11 | Oligonucleotides used in this paper.

Herzog, M., Dorne, A. M., and Grellet, F. (1995). GASA, a gibberellin-regulated gene family from Arabidopsis thaliana related to the tomato GAST1 gene. Plant Mol. Biol. 27, 743-752. doi: 10.1007/BF00020227

Holappa, L. D., and Walker-Simmons, M. K. (1995). The wheat abscisic acidresponsive protein kinase mRNA, PKABA1, is up-regulated by dehydration, cold temperature, and osmotic stress. Plant Physiol. 108, 1203-1210.

Hrabak, E. M., Chan, C. W. M., Gribskov, M., Harper, J. F., Choi, J. H., Halford, N., et al. (2003). The Arabidopsis CDPK-SnRK superfamily of protein kinases. Plant Physiol. 132, 666-680. doi: 10.1104/pp.102.011999

Huai, J. L., Wang, M., He, J., Zheng, J., Dong, Z., Lv, H., et al. (2008). Cloning and characterization of the SnRK2 gene family from Zea mays. Plant Cell Rep. 27, 1861-1868. doi: 10.1007/s00299-008-0608-8

Kobayashi, Y., Yamamoto, S., Minami, H., Kagaya, Y., and Hattori, T. (2004). Differential activation of the rice sucrose nonfermenting1-related protein kinase 2 family by hyperosmotic stress and abscisic acid. Plant Cell 16, 1163-1177. doi: 10.1105/tpc.019943

Kubo, M., Udagawa, M., Nishikubo, N., Horiguchi, G., Yamaguchi, M., Ito, J., et al. (2005). Transcription switches for protoxylem and metaxylem vessel formation. Genes Dev. 19, 1855-1860. doi: 10.1101/gad.1331305

Leung, J., Bouvier-Durand, M., Morris, P. C., Guerrier, D., Chefdor, F., and Giraudat, J. (1994). Arabidopsis ABA response gene ABI1: features of a calcium-modulated protein phosphatase. Science 264, 1448-1452. doi: 10.1126/science.7910981

Leung, J., Merlot, S., and Giraudat, J. (1997). The Arabidopsis ABSCISIC ACID-INSENSITIVE 2 (ABI2) and ABI1 genes encode homologous protein phosphatases 2C involved in abscisic acid signal transduction. Plant Cell 9, 759-771. doi: 10.1105/tpc.9.5.759

Mao, X., Zhang, H., Tian, S., Chang, X., and Jing, R. (2010). TaSnRK2.4, an SNF1type serine/threonine protein kinase of wheat (Triticum aestivum L.), confers enhanced multistress tolerance in Arabidopsis. J. Exp. Bot. 61, 683-696. doi: 10.1093/jxb/erp331

Meyer, K., Leube, M. P., and Grill, E. (1994). A protein phosphatase 2C involved in ABA signal transduction in Arabidopsis thaliana. Science 264, 1452-1455. doi: 10.1126/science. 8197457

Mi, H., Muruganujan, A., Casagrande, J. T., and Thomas, P. D. (2013). Large-scale gene function analysis with the PANTHER classification system. Nat. Protoc. 8, 1551-1566. doi: 10.1038/nprot.2013.092

Mizoguchi, M., Umezawa, T., Nakashima, K., Kidokoro, S., Takasaki, H., Fujita, Y., et al. (2010). Two closely related subclass II SnRK2 protein kinases cooperatively regulate drought-inducible gene expression. Plant Cell Physiol. 51, 842-847. doi: 10.1093/pcp/pcq041

Nakamichi, N., Kusano, M., Fukushima, A., Kita, M., Ito, S., Yamashino, T., et al. (2009). Transcript profiling of an Arabidopsis PSEUDO RESPONSE REGULATOR arrhythmic triple mutant reveals a role for the circadian clock in cold stress response. Plant Cell Physiol. 50, 447-462. doi: 10.1093/pcp/pcp004

Nakashima, K., Fujita, Y., Kanamori, N., Katagiri, T., Umezawa, T., Kidokoro, S., et al. (2009). Three Arabidopsis SnRK2 protein kinases, SRK2D/SnRK2.2, SRK2E/SnRK2.6/OST1 and SRK2I/SnRK2.3, involved in ABA signaling are essential for the control of seed development and dormancy. Plant Cell Physiol. 50, 1345-1363. doi: 10.1093/pcp/pcp083 
Ng, L. M., Melcher, K., Teh, B. T., and Xu, H. E. (2014). Abscisic acid perception and signaling: structural mechanisms and applications. Acta Pharmacol. Sin. 35, 567-584. doi: 10.1038/aps.2014.5

Ng, L. M., Soon, F. F., Zhou, X. E., West, G. M., Kovach, A., Suino-Powell, K. M., et al. (2011). Structural basis for basal activity and autoactivation of abscisic acid (ABA) signaling SnRK2 kinases. Proc. Natl. Acad. Sci. U.S.A. 108, 21259-21264. doi: $10.1073 /$ pnas.1118651109

Nishimura, N., Yoshida, T., Kitahata, N., Asami, T., Shinozaki, K., and Hirayama, T. (2007). ABA-Hypersensitive Germination 1 encodes a protein phosphatase $2 \mathrm{C}$, an essential component of abscisic acid signaling in Arabidopsis seed. Plant J. 50, 935-949. doi: 10.1111/j.1365-313X.2007.03107.x

Ohtani, M., Demura, T., and Sugiyama, M. (2013). Arabidopsis ROOT INITIATION DEFECTIVE1, a DEAH-Box RNA helicase involved in premRNA splicing, is essential for plant development. Plant Cell 25, 2056-2069. doi: $10.1105 /$ tpc.113.111922

Ohtani, M., Nishikubo, N., Xu, B., Yamaguchi, M., Mitsuda, N., Goué, N., et al. (2011). A NAC domain protein family contributing to the regulation of wood formation in poplar. Plant J. 67, 499-512. doi: 10.1111/j.1365313X.2011.04614.x

Porra, R. J., Thompson, W. A., and Kriedemann, P. E. (1989). Determination of accurate extinction coefficients and simultaneous equations for assaying chlorophylls a and b extracted with four different solvents: verification of the concentration of chlorophyll standards by atomic absorption spectroscopy. Biochim. Biophys. Acta Bioenerget. 975, 384-394. doi: 10.1016/S00052728(89)80347-0

Saez, A., Apostolova, N., Gonzalez-Guzman, M., Gonzalez-Garcia, M. P., Nicolas, C., Lorenzo, O., et al. (2004). Gain-of-function and loss-of-function phenotypes of the protein phosphatase $2 \mathrm{C} \mathrm{HAB1}$ reveal its role as a negative regulator of abscisic acid signalling. Plant J. 37, 354-369. doi: 10.1046/j.1365313X.2003.01966.x

Sherson, S. M., Alford, H. L., Forbes, S. M., Wallace, G., and Smith, S. M. (2003). Roles of cell-wall invertases and monosaccharide transporters in the growth and development of Arabidopsis. J. Exp. Bot. 54, 525-531. doi: $10.1093 / \mathrm{jxb} / \mathrm{erg} 055$

Shin, R., Alvarez, S., Burch, A. Y., Jez, J. M., and Schachtman, D. P. (2007). Phosphoproteomic identification of targets of the Arabidopsis sucrose nonfermenting-like kinase SnRK2. 8 reveals a connection to metabolic processes. Proc. Natl. Acad. Sci. U.S.A. 104, 6460-6465. doi: 10.1073/pnas.0610208104

Song, X. Q., Ohtani, M., Hori, C., Takebayasi, A., Hiroyama, R., Rejab, N. A., et al. (2015). Physical interaction between SnRK2 and PP2C is conserved in Populus trichocarpa. Plant Biotechnol. 32, 337-341. doi: 10.5511/plantbiotechnology.15.0813b

Sopory, S. K., and Munshi, M. (1998). Protein kinases and phosphatases and their role in cellular signaling in plants. Crit. Rev. Plant Sci. 17, 245-318. doi: $10.1080 / 07352689891304230$

Tian, S., Mao, X., Zhang, H., Chen, S., Zhai, C., Yang, S., et al. (2013). Cloning and characterization of TaSnRK2.3, a novel SnRK2 gene in common wheat. J. Exp. Bot. 64, 2063-2080. doi: 10.1093/jxb/ert072
Umezawa, T., Nakashima, K., Miyakawa, T., Kuromori, T., Tanokura, M., Shinozaki, K., et al. (2010). Molecular basis of the core regulatory network in ABA responses: sensing, signaling and tranport. Plant Cell Physiol. 51, 1821-1839. doi: 10.1093/pcp/pcq156

Umezawa, T., Sugiyama, N., Mizoguchi, M., Hayashi, S., Myouga, F., YamaguchiShinozaki, K., et al. (2009). Type 2C protein phosphatases directly regulate abscisic acid-activated protein kinases in Arabidopsis. Proc. Natl. Acad. Sci. U.S.A. 106, 17588-17593. doi: 10.1073/pnas.0907095106

Umezawa, T., Sugiyama, N., Takahashi, F., Anderson, J. C., Ishihama, Y., Peck, S. C., et al. (2013). Genetics and phosphoproteomics reveal a protein phosphorylation network in the abscisic acid signaling pathway in Arabdiopsis thaliana. Sci. Signal 6:rs8 doi: 10.1126/scisignal.2003509

Umezawa, T., Yoshida, R., Maruyama, K., Yamaguchi-Shinozaki, K., and Shinozaki, K. (2004). SRK2C, a SNF1-related protein kinase 2, improves drought tolerance by controlling stress-responsive gene expression in Arabidopsis thaliana. Proc. Natl. Acad. Sci. U.S.A. 101, 17306-17311. doi: 10.1073/pnas.0407758101

Vilela, B., Moreno, A., Capellades, M., Pagès, M., and Lumbreras, V. (2012). $\mathrm{ZmSnRK} 2.8$ responds to ABA through the SnRK2-PP2C complex. Maydica 57, $11-18$.

Vlad, F., Rubio, S., Rodrigues, A., Sirichandra, C., Belin, C., Robert, N., et al. (2009). Protein phosphatases 2C regulate the activation of the Snfl-related kinase OST1 by abscisic acid in Arabidopsis. Plant Cell 21, 3170-3184. doi: 10.1105/tpc.109.069179

Yoshida, R., Hobo, T., Ichimura, K., Mizoguchi, T., Takahashi, F., Aronso, J., et al. (2002). ABA-activated SnRK2 protein kinase is required for dehydration stress signaling in Arabidopsis. Plant Cell Physiol. 43, 1473-1483. doi: $10.1093 / \mathrm{pcp} / \mathrm{pcf} 188$

Zhang, H., Mao, X., Jing, R., Chang, X., and Xie, H. (2011). Characterization of a common wheat (Triticum aestivum L.) TaSnRK2.7 gene involved in abiotic stress reponses. J. Exp. Bot. 62, 975-988. doi: 10.1093/jxb/erq328

Zhang, H., Mao, X., Wang, C., and Jing, R. (2010). Overexpression of a common wheat gene TaSnRK2.8 enhances tolerance to drought, salt and low temperature. PLOS ONE 5:e16041. doi: 10.1371/journal.pone. 0016041

Zhang, J., Feng, J., Lu, J., Yang, Y., Zhang, X., Wan, D., et al. (2014). Transcriptome differences between two sister desert poplar species under salt stress. BMC Genomics 15:337. doi: 10.1186/1471-2164-15-337

Conflict of Interest Statement: The authors declare that the research was conducted in the absence of any commercial or financial relationships that could be construed as a potential conflict of interest.

Copyright (c) 2016 Song, Yu, Hori, Demura, Ohtani and Zhuge. This is an open-access article distributed under the terms of the Creative Commons Attribution License (CC $B Y)$. The use, distribution or reproduction in other forums is permitted, provided the original author(s) or licensor are credited and that the original publication in this journal is cited, in accordance with accepted academic practice. No use, distribution or reproduction is permitted which does not comply with these terms. 